\title{
Sistema de indicadores de gestión para pymes, sector metalmecánico
}

\section{(System of performance indicators for SMEs, metalworking sector)}

\author{
Cristina Viteri Sánchez ${ }^{1}$, Jorge Viteri Moya ${ }^{2}$, Edison Matute Déleg ${ }^{3}$
}

\begin{abstract}
Resumen:
El presente trabajo se centró en el diseño y propuesta de indicadores de gestión para las pequeñas y medianas industrias (pymes) metalmecánicas en la ciudad de Quito. Se identificó el número de metalmecánicas para el estudio. Mediante análisis se conoció el funcionamiento de las empresas y se determinó qué áreas merecen ser permanentemente monitoreadas, para ello se empleó herramientas como el análisis DAFO, entrevistas y reuniones con los gerentes de las empresas, cuyos resultados demandaron el planteamiento de una estrategia genérica, que abarcó la formulación de la misión, visión, y valores corporativos. Se establecieron objetivos y metas, estos fueron clasificados de acuerdo a las cuatro perspectivas del Cuadro de Mando Integral. Luego se procedió a determinar los indicadores que permitan medir el funcionamiento correcto de la estrategia a emplearse. Se propusieron 10 indicadores de gestión, de acuerdo al análisis de la realidad de las metalmecánicas. Se definieron 3 indicadores financieros, 2 indicadores enfocados al cliente, 3 indicadores para controlar los procesos internos y 2 direccionados a la perspectiva de formación y crecimiento. La utilización de estos indicadores es la clave del desempeño y el control de procesos en cada metalmecánica.
\end{abstract}

Palabras clave: Indicadores; pymes; sector metalmecánico; estrategia; Cuadro de Mando Integral.

\begin{abstract}
:
This paper focused on the design and proposal of management indicators for small and medium metalworking enterprises (SMEs) in Quito. Metalworking number was established for the study. It was met and determined which areas deserve to be constantly monitored, by analyzing the performance of the companies, for this purpose, it was used a SWOT analysis, interviews and meetings with company managers, the results demanded the approach of a generic strategy, which included the formulation of the mission, vision and corporate values. Objectives and goals were established, they were classified according to the four perspectives of the Balanced Scorecard. Then it was possible to identify indicators to measure the proper functioning of the strategy proposed. 10 indicators of management were developed, according to the analysis of the reality of metalworking enterprises. So it was determined 3 financial indicators, 2 indicators focused on customers, 3 indicators for monitor internal business processes and 2 addressed the prospect of learning and growth. The use of these indicators is the key of performance and process control in each metalworking company.
\end{abstract}

Keywords: Indicators; SMEs; metalworking sector; strategy; Balanced Scorecard.

\footnotetext{
${ }^{1}$ Universidad Tecnológica Equinoccial, Dirección de Vinculación con la Colectividad, Quito-Ecuador (vscb30768@ute.edu.ec).

2 Universidad Tecnológica Equinoccial, Decanato de la Facultad de Ciencias de la Ingeniería, QuitoEcuador (jviteri@ute.edu.ec).

${ }^{3}$ Universidad Tecnológica Equinoccial, Supervisión de Talleres-Facultad de Ciencias de la Ingeniería, Quito-Ecuador (mdeb34579@ute.edu.ec).
} 


\section{Introducción}

Todo tipo de empresa u organización debe su éxito y continuidad a la capacidad que ella tenga para medir y evaluar sus objetivos estratégicos, desde una perspectiva financiera, de procesos, del cliente y nivel de aprendizaje (Marquéz, 2006). Esto es posible con el uso de indicadores de gestión establecidos de forma adecuada, mediante estudios previos de la realidad de las empresas.

El presente trabajo se enfoca en el estudio de las pymes metalmecánicas en Quito, con el apoyo del Colegio de Ingenieros Mecánicos de Pichincha (CIMEPI), ente que brinda ayuda técnica a los ingenieros mecánicos de la provincia, se ha convenido en la necesidad primordial del desarrollo de un sistema genérico de indicadores, que sirvan para medir la eficiencia y eficacia de las actividades y procesos, así como el control del cumplimiento de la estrategia de las empresas. La mejora de la gestión del sector metalmecánico se ha convertido en prioridad, ya que de acuerdo al CIMEPI, dicha área de la producción es uno de los ejes primordiales para el desarrollo económico del país.

Las pequeñas y medianas empresas, más conocidas por su acrónimo: pymes, es un término que apareció en la segunda mitad del siglo XX, época en la que también se empezó a segmentar a las empresas por su número de empleados, facturación anual, activos, etc. (Castañeda, 2009).

Los países industrializados, emergentes y en desarrollo han experimentado el constante crecimiento de las pymes, debido a la sinergia ejercida por personas, familias y empresas, que con su esfuerzo y actitud han sabido canalizar la generación de empleo y demanda creciente de productos y servicios, esto ha convertido a las pymes en factores indispensables para el avance de la economía de los países (Corporación EKOS, 2010).

De acuerdo a la información proporcionada por el Servicio de Rentas Internas (SRI), en 2011 existieron 27646 pymes, donde 13332 pertenecen al sector metalmecánico, divididas en 4661 medianas $(34,96 \%)$ y 8671 pequeñas $(65,04 \%)$ (EKOS Negocios, 2012$)$.

La planeación no es exclusiva de las compañías grandes, las pymes necesitan de ella tanto como las grandes empresas, es posible que en mayor medida, ya que su supervivencia se encuentra siempre en constante peligro (Scali \& Tapia, 2011).

Las pymes que no se preparan, no evalúan las necesidades y expectativas de los consumidores y confían en actores externos sin experiencia, fracasan en ampliar los mercados. La asociatividad es requisito fundamental para el desarrollo de este importante sector de la industria (Universidad Andina Simón Bolívar, 2012).

Por otra parte, el área de la metalmecánica ha logrado un crecimiento moderado durante los últimos cinco años. Según la Cámara de la Pequeña Industria de Pichincha, el crecimiento 
económico en el período 2007 - 2011 es de entre el 2\% y el 3\%. La falta de capacitación, de crédito y de impulso a la cadena productiva metalmecánica son algunos de los problemas que ha tenido que enfrentar este sector (El Comercio, 2012).

El sector de la metalmecánica abarca una gran diversidad de actividades productivas, que van desde la fundición a la transformación y soldadura así como también al tratamiento químico de diferentes superficies (CORPEI, 2009).

Finalmente, es preciso indicar que la aplicación de indicadores de gestión en los procesos productivos de empresas metalmecánicas reducirá significativamente el riesgo de no cumplir con la misión y visión de las organizaciones, gracias al adecuado control durante toda la cadena de valor (Heredia, 2001).

Con estos antecedentes, el presente proyecto busca establecer un Cuadro de Mando Integral basado en los conceptos que establecieron Kaplan y Norton, donde las empresas son capaces de idear estrategias, a la vez que miden su desempeño al usar indicadores. Con esto se obtendrá una serie de medidas, que serán aplicadas a la empresa metalmecánica, de manera que se establezca un programa de gestión y control permanente (Kaplan \& Norton, 2009).

\section{Metodología}

El presente estudio se desarrolló en tres etapas:

- Análisis de la situación actual de las pymes metalmecánicas.

La investigación se enmarcó en la elaboración de una propuesta factible que cubra las necesidades de las metalmecánicas. Éstas fueron evidenciadas a través de una investigación de campo. Dicha investigación se caracterizó por el análisis in situ de la realidad de las metalmecánicas; además se obtuvo información mediante encuestas, reuniones y entrevistas con los gerentes y trabajadores de los talleres.

Luego de la visita a las empresas, se pudo analizar la situación actual en la que se encuentran las metalmecánicas, mediante la identificación de las debilidades y fortalezas, con el empleo de la matriz de evaluación de factores internos (EFI). Asimismo el análisis externo relacionado con las amenazas y oportunidades fue posible con el desarrollo de la matriz de evaluación de factores externos (EFE).

- Determinación de objetivos y metas estratégicas para las empresas metalmecánicas con base en las cuatro perspectivas del Cuadro de Mando Integral. 
La información obtenida de la evaluación de factores tanto internos como externos sirvió también para la elaboración del análisis DAFO (debilidades, amenazas, fortalezas y oportunidades), de donde se consiguió definir las iniciativas estratégicas para las empresas metalmecánicas.

Mediante reuniones de grupo con los gerentes de las metalmecánicas, las estrategias resultantes del análisis DAFO fueron socializadas y en base a las cuatro perspectivas del Cuadro de Mando Integral, se pudieron identificar los objetivos y metas estratégicas, que posteriormente fueron relacionadas mediante el uso del modelo causa-efecto.

Asimismo con la identificación de los procesos clave para la consecución de los objetivos planteados, fue posible determinar y estructurar la cadena de valor para las empresas metalmecánicas.

Antes del diseño de los indicadores de gestión, fue necesaria la restructuración y clarificación de la misión, visión y valores corporativos de las empresas metalmecánicas. Para esto se elaboraron reuniones con los gerentes de las metalmecánicas, donde fue posible llegar a consensos en cuanto a la estrategia de las pymes metalmecánicas.

- Elaboración de un sistema de indicadores de gestión para controlar y evaluar los objetivos planteados de acuerdo a las perspectivas del Cuadro de Mando Integral.

Una vez definida la misión, visión, valores corporativos, los objetivos y metas estratégicas, el pensamiento estratégico para las pymes metalmecánicas quedó plasmado y debidamente socializado mediante técnicas de trabajo en grupo. El siguiente paso fue el diseño de un sistema de indicadores que aseguren la correcta ejecución de las estrategias y la evaluación de la medida en que las metas estratégicos están siendo cumplidas.

Apoyados en las cuatro perspectivas del cuadro de mando integral, el grupo de indicadores establecido buscó la forma de traducir operativamente los objetivos planteados así como determinar su correcto funcionamiento.

De todos los indicadores posibles de diseñar, se tomaron en cuenta aquellos alineados a la misión y visión que se desarrolló, de manera que faciliten el monitoreo de áreas estratégicas (cadena de valor) y proporcionen a los gerentes elementos de juicio para tomar las mejores decisiones tendientes a optimizar recursos y mejorar la competitividad de la empresa.

\section{Resultados y Discusión}

Para el cálculo del tamaño de la muestra se consideraron algunas propiedades como el error máximo permisible, y quedó fijado en un $15 \%$, debido a que se trata de un primer estudio de 
metalmecánicas, con el apoyo del CIMEPI. Como en este caso se conoce el tamaño de la población, se aplicó la siguiente fórmula (Reyes, 2011):

$$
n=\frac{Z^{2} p q N}{N E^{2}+Z^{2} p q}
$$

Donde:

$\mathrm{n}$ : es el tamaño de la muestra

Z: es el nivel de confianza

p: es la variabilidad positiva

q: es la variabilidad negativa

$\mathrm{N}$ : es el tamaño de la población

E: es la precisión o el error

Así se tiene:

$$
\begin{gathered}
n=\frac{\left(1.96^{2}\right)(0.5)(0.5)(120)}{(120)\left(0.15^{2}\right)+\left(1.96^{2}\right)(0.5)(0.5)} \\
n=31
\end{gathered}
$$

El cálculo del tamaño de la muestra indicó que se debían visitar a 31 empresas metalmecánicas ubicadas en la ciudad de Quito y los valles, donde se obtuvo información directamente de los propietarios o encargados de los talleres mediante encuestas y entrevistas.

En la Tabla 1 se puede observar a manera de resumen cómo las 31 metalmecánicas estudiadas trabajan, basado en las perspectivas del cuadro de mando integral.

Tabla 1. Situación de las metalmecánicas estudiadas con respecto a las perspectivas de cuadro de mando integral

\begin{tabular}{|c|l|}
\hline PERSPECTIVAS & \multicolumn{1}{|c|}{ UTILIZACIÓN EN LAS METALMECÁNICAS } \\
\hline Financiera & $\begin{array}{l}12 \text { metalmecánicas utilizan el total de ventas producidas reflejadas en el índice de } \\
\text { utilidades como un indicador de gestión. }\end{array}$ \\
\hline Del cliente & $\begin{array}{l}\text { Las metalmecánicas que utilizan a la satisfacción del cliente como indicador, la determinan } \\
\text { en el momento de la entrega del producto. Se la realiza de forma empírica al observar la } \\
\text { reacción del cliente luego de receptar el pedido, es así que los propietarios se quedan con } \\
\text { una percepción que puede ser errónea. Tan solo cuatro metalmecánicas aplican encuestas } \\
\text { de satisfacción. }\end{array}$ \\
\hline De los procesos internos & $\begin{array}{l}\text {-Los propietarios y gerentes de las metalmecánicas confunden a los parámetros de } \\
\text { proceso con indicadores, por ejemplo; temperaturas, medidas de longitud y datos de } \\
\text { control dados automáticamente por máquinas. }\end{array}$ \\
& $\begin{array}{l}\text {-En ninguna empresa metalmecánica se controlan tiempos en los procesos, ni se } \\
\text { establecen métodos de trabajo específicos (estandarización). }\end{array}$ \\
\hline Crecimiento y Desarrollo & $\begin{array}{l}- \text { A simple vista se pudo identificar las malas condiciones en la que se encuentran los } \\
\text { talleres metalmecánicos y la falta de orden, además los equipos y máquinas no cuentan } \\
\text { con el mantenimiento adecuado. } \\
\text {-En ninguna empresa metalmecánica existen planes de capacitación para el personal, se } \\
\text { confía en lo que dicta la experiencia de los trabajadores y no se toma en cuenta el } \\
\text { desarrollo cognitivo de los trabajadores y tampoco existen incentivos como la oportunidad } \\
\text { de crecimiento para los trabajadores. No se trabaja en el tema de innovación. }\end{array}$ \\
\hline
\end{tabular}


El $40 \%$ de las pymes metalmecánicas estudiadas cuentan con visión, misión y valores corporativos establecidos. Todos ellos han sido propuestos por parte de los gerentes 0 propietarios, pero su socialización no ha sido la adecuada.

Se desarrolló una misión y visión genérica, de modo que puedan ser aplicables a todas las metalmecánicas estudiadas, ya que sus realidades son parecidas. Así la misión propuesta fue:

"Entregar al Ecuador soluciones integrales a necesidades y requerimientos del área metalmecánica a través de la fabricación y comercialización de estructuras y piezas con altos estándares de calidad, cultivando máximos valores corporativos para generar beneficios a nuestros proveedores, empleados, clientes y accionistas".

La visión quedó de la siguiente manera:

"Ser empresas líderes a nivel nacional en la fabricación y comercialización de productos metalmecánicos, asegurando la entrega de productos y servicios de calidad a nuestros clientes, trabajando con calidad, responsabilidad, ética, y amabilidad, para la generación de rentabilidad, confianza y satisfacción a nuestros colaboradores".

Finalmente debido a la falta de valores corporativos que apoyen al pensamiento estratégico, se establecieron valores y principios como: responsabilidad, ética, calidad, respeto, trabajo en equipo, y satisfacción al cliente.

La usar las técnicas de observación directa y reuniones con los gerentes y administradores de las pymes metalmecánicas, fue posible la determinación de las debilidades y fortalezas, así como de amenazas y oportunidades, cuyos resultados pueden ser observados en las tablas 2 y 3 respectivamente. Este análisis fue realizado una vez que se definieron la visión, misión y valores corporativos.

Tabla 2. Debilidades y fortalezas

\begin{tabular}{|l|l|}
\hline \multirow{5}{*}{ Debilidades } & -Las empresas no cuentan con indicadores de gestión, con base en las cuatro \\
& perspectivas del CMI. \\
& -Los objetivos estratégicos no están alineados con la misión y visión. \\
& -No cuentan con estrategias de crecimiento e innovación. \\
& -Ausencia de un control financiero efectivo. \\
& -Retardo en la entrega de productos. \\
& -Los objetivos y metas no están formalmente definidos. \\
\hline \multirow{5}{*}{ Fortalezas } & -Confianza de los clientes \\
& -Las empresas tienen una estructura jerárquica flexible \\
& -Precios bajos en comparación con las grandes empresas \\
& -Atención personalizada al cliente \\
& -Flexibilidad operacional \\
& -Para que se realice el servicio no se exigen grandes trámites \\
& -Liderazgo gerencial claramente definido \\
& -Estabilidad laboral \\
\hline
\end{tabular}


Tabla 3. Oportunidades y amenazas

\begin{tabular}{|l|l|}
\hline \multirow{5}{*}{ Oportunidades } & $\begin{array}{l}\text {-Mercado en crecimiento } \\
\text {-Los avances tecnológicos no suponen retraso en los procesos, debido a la gran } \\
\text { cantidad de operaciones manuales } \\
\text {-Nuevos enfoques empresariales que coadyuven a la satisfacción del cliente } \\
\text {-Poca competencia } \\
\text {-Diseño e innovación de productos }\end{array}$ \\
\hline Amenazas & $\begin{array}{l}\text {-Demoras en la entrega de productos terminados } \\
\text {-Captación de clientes por parte de la competencia } \\
\text {-Equipos de alta tecnología solo disponible para grandes empresas por los altos } \\
\text { costos de operación } \\
\text { - Poca oferta de capacitaciones en el área metalmecánica }\end{array}$ \\
\hline
\end{tabular}

Una vez hecho el análisis tanto interno como externo de las pymes metalmecánicas se realizó el análisis DAFO (tabla 4), de manera que fue posible determinar estrategias que sirvan para lograr objetivos a largo plazo.

Tabla 4. Análisis DAFO

\begin{tabular}{|c|c|c|}
\hline $\begin{array}{l}\text { ANÁLISIS } \\
\text { EXTERNO }\end{array}$ & $\begin{array}{l}\quad \text { OPORTUNIDADES } \\
\text {-Mercado en crecimiento } \\
\text {-Confianza de los clientes } \\
\text { - Los avances tecnológicos no } \\
\text { suponen retraso en los procesos, } \\
\text { debido a la gran cantidad de } \\
\text { operaciones manuales } \\
\text { - La tecnología ayudaría a } \\
\text { entregar productos de mejor } \\
\text { calidad } \\
\text { - Nuevos enfoques de las } \\
\text { empresas a la satisfacción del } \\
\text { cliente } \\
\text {-Alto costo en los productos de la } \\
\text { competencia }\end{array}$ & $\begin{array}{l}\quad \text { AMENAZAS } \\
\text {-Demoras en la entrega de } \\
\text { materiales y suministros } \\
\text {-Captación de clientes por parte de la } \\
\text { competencia } \\
\text { - Equipos de alta tecnología solo } \\
\text { disponible para grandes empresas } \\
\text { - Poca oferta de capacitaciones en el } \\
\text { área metalmecánica }\end{array}$ \\
\hline $\begin{array}{l}\text { FORTALEZAS } \\
\text {-Cuentan con misión, visión y } \\
\text { valores claramente definidos } \\
\text {-Las empresas tienen una } \\
\text { estructura organizativa flexible } \\
\text {-Precios adecuados de los } \\
\text { productos y servicios } \\
\text {-Atención al cliente personalizada } \\
\text {-Para que se realice el servicio no } \\
\text { exige grandes trámites } \\
\text {-Liderazgo claramente definido } \\
\text {-Estabilidad laboral }\end{array}$ & $\begin{array}{l}\text { ESTRATEGIAS OFENSIVAS } \\
\text { (FO) } \\
\text {-Implementación de estrategias } \\
\text { de marketing } \\
\text {-Elaboración de portafolio de } \\
\text { clientes reales y potenciales } \\
\text {-Ofrecer al mercado nacional un } \\
\text { portafolio mayor de productos, } \\
\text { con promoción de la innovación. }\end{array}$ & $\begin{array}{l}\text { ESTRATEGIAS DEFENSIVAS (FA) } \\
\text {-Implementar un sistema de } \\
\text { evaluación de proveedores críticos } \\
\text {-Desarrollar un sistema de cobranzas } \\
\text { eficaz }\end{array}$ \\
\hline \begin{tabular}{l}
\multicolumn{1}{c}{ DEBILIDADES } \\
-Las empresas no cuentan con \\
indicadores de gestión \\
-Los objetivos estratégicos no \\
están alineados con la misión y \\
visión \\
- No cuentan con estrategias de \\
crecimiento \\
-Ausencia de un control financiero \\
efectivo \\
-Tiempo de entrega de productos \\
elevado \\
-Los objetivos y metas no están \\
formalmente definidos
\end{tabular} & $\begin{array}{l}\text { ESTRATEGIAS ADAPTATIVAS } \\
\text { (DO) } \\
\text {-Llevar a cabo un plan de mejora } \\
\text { de la calidad } \\
\text {-Desarrollar un plan de } \\
\text { capacitación al personal }\end{array}$ & $\begin{array}{l}\quad \text { ESTRATEGIAS DE } \\
\text { SUPERVIVENCIA (DA) } \\
\text {-Establecer el control de gestión } \\
\text { mediante indicadores } \\
\text {-Mejorar la relación con los clientes } \\
\text {-Determinar programas económicos } \\
\text { para mejorar la infraestructura de los } \\
\text { talleres y renovación de maquinaria } \\
\text {-Medir la calidad de servicio } \\
\text {-Elaborar un plan de acción para } \\
\text { responder a reclamos de clientes } \\
\text {-Establecer un programa de } \\
\text { reducción de costos operativos }\end{array}$ \\
\hline
\end{tabular}


Luego de conocer la realidad de las empresas metalmecánicas gracias al análisis DAFO, fue necesario establecer objetivos, de modo que los principales problemas que enfrentan sean cubiertos por estrategias, que permitan que mejore la situación de este sector industrial.

Con base en las perspectivas del cuadro de mando integral se ha procedido a establecer 10 objetivos estratégicos, se presentan en la tabla 5:

Tabla 5. Objetivos Estratégicos de la empresa bajo las perspectivas del CMI

\begin{tabular}{|c|l|}
\hline Perspectiva & \multicolumn{1}{c|}{ Objetivos estratégicos } \\
\hline Financiera & $\begin{array}{l}\text {-Disminuir los costos de producción } \\
\text {-Tecnificar el sistema de cobro } \\
\text {-Incrementar la rentabilidad de la empresa }\end{array}$ \\
\hline Del cliente & $\begin{array}{l}\text {-Acrecentar el grado de satisfacción del cliente } \\
\text {-Incrementar el número de clientes }\end{array}$ \\
\hline Procesos internos & -Establecer un plan de calidad total \\
& -Disminuir los tiempos de entrega del producto demandado \\
& -Estandarizar los procesos \\
\hline Crecimiento y desarrollo & -Generar planes de adiestramiento y capacitación \\
& -Fomentar procesos de innovación \\
\hline
\end{tabular}

La figura 1 determina la relación establecida entre los objetivos estratégicos dentro de la organización mediante el esquema del modelo causa efecto.

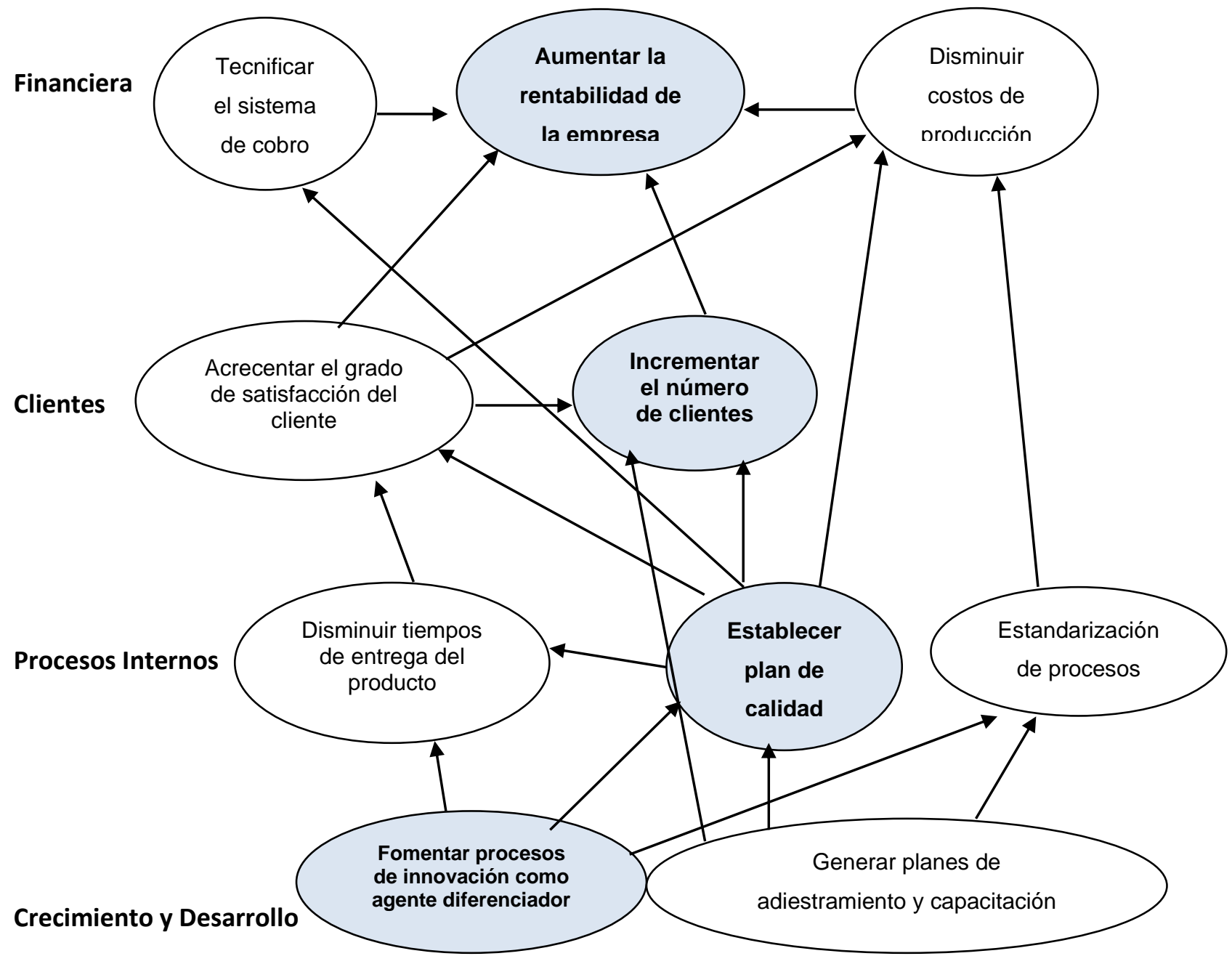

Figura 1. Modelo causa efecto entre los objetivos estratégicos de la empresa 
Una vez que se realizó la figura causa-efecto, se pudo establecer que los objetivos de mayor impacto crítico para el desarrollo de la estrategia fueron: aumentar la rentabilidad e incrementar el número de clientes. Se definió que para lograr este cometido es necesario establecer un plan de calidad integral y es indispensable el fomento de procesos de innovación ajustándolo a la realidad tecnológica de las pymes estudiadas.

La figura 2 representa las actividades que generan valor para las empresas metalmecánicas.

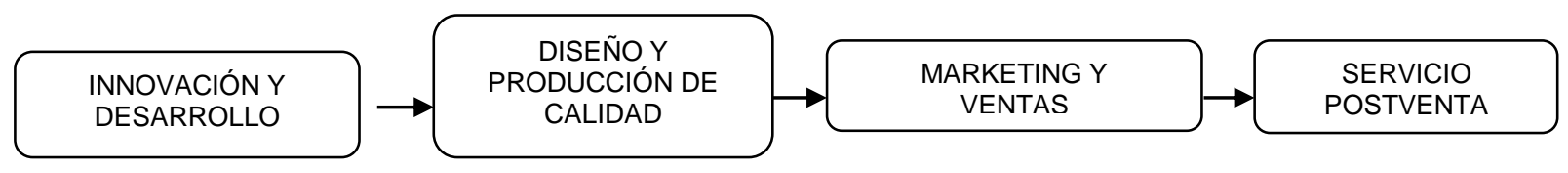

Figura 2. Cadena de valor de las empresas metalmecánicas

Una vez que se establecieron los objetivos estratégicos y la relación entre ellos a través del modelo causa-efecto, así como el establecimiento de la cadena de valor, se procedió a definir las estrategias, mismas que se presentan en la tabla 6.

Tabla 6. Establecimiento de estrategias a partir de los objetivos estratégicos de las empresas metalmecánicas.

\begin{tabular}{|c|c|c|}
\hline Perspectiva & Objetivo Estratégico & Estrategia \\
\hline \multirow{3}{*}{ Financiera } & $\begin{array}{l}\text { Disminuir los costos de } \\
\text { producción }\end{array}$ & \multirow{3}{*}{$\begin{array}{l}\text {-Establecer un plan estratégico alineado al concepto } \\
\text { estratégico de la empresa, que contemple un sistema } \\
\text { de indicadores definido en base a las perspectivas } \\
\text { del CMl. } \\
\text {-Lograr el establecimiento de un sistema de } \\
\text { cobranzas, que reflejen el volumen de ventas y } \\
\text { faciliten el pago de impuestos. }\end{array}$} \\
\hline & Tecnificar el sistema de cobro & \\
\hline & Incrementar la rentabilidad & \\
\hline \multirow[b]{2}{*}{ De los Clientes } & $\begin{array}{l}\text { Acrecentar el grado de } \\
\text { satisfacción del cliente }\end{array}$ & \multirow{2}{*}{$\begin{array}{l}\text {-Desarrollar un programa de acción para incrementar } \\
\text { la satisfacción de los clientes a través de la oferta de } \\
\text { productos de calidad, consecución de cero demoras } \\
\text { en los tiempos de entrega, establecimiento de costos } \\
\text { competitivos, y un trato efectivo y directo con los } \\
\text { líderes de la organización. } \\
\text {-Implementación de estrategias de marketing. } \\
\text {-Elaboración de portafolio de clientes reales y } \\
\text { potenciales. } \\
\text {-Plan de acción para respuesta inmediata ante } \\
\text { reclamos de clientes. }\end{array}$} \\
\hline & $\begin{array}{l}\text { Incrementar el número de } \\
\text { clientes }\end{array}$ & \\
\hline \multirow{3}{*}{$\begin{array}{l}\text { De los } \\
\text { Procesos } \\
\text { Internos }\end{array}$} & $\begin{array}{c}\text { Establecer calidad total en los } \\
\text { procesos }\end{array}$ & \multirow{3}{*}{$\begin{array}{l}\text {-Aplicar un plan de control de calidad total por un } \\
\text { período de } 3 \text { años. } \\
\text {-Definir y formalizar métodos de trabajo, incluyendo el } \\
\text { control de tiempos para todos los procesos. }\end{array}$} \\
\hline & $\begin{array}{l}\text { Disminuir tiempo de entrega del } \\
\text { producto }\end{array}$ & \\
\hline & Estandarización de procesos & \\
\hline \multirow{2}{*}{$\begin{array}{l}\text { De Formación } \\
\text { y Crecimiento }\end{array}$} & $\begin{array}{l}\text { Diseño de productos y/o } \\
\text { procesos innovadores }\end{array}$ & \multirow{2}{*}{$\begin{array}{l}\text {-Fomentar el trabajo en equipo, la motivación del } \\
\text { trabajador y el uso de plataformas tecnológicas para } \\
\text { la investigación. } \\
\text {-Diseñar programas de capacitación permanente para } \\
\text { el personal. }\end{array}$} \\
\hline & Generar planes de capacitación & \\
\hline
\end{tabular}

En las tablas 7, 8, 9 y 10 se pueden apreciar los indicadores de gestión establecidos para las empresas metalmecánicas. Se tomó en cuenta cada perspectiva del Cuadro de Mando Integral. La frecuencia de monitoreo se estableció de dos maneras: de forma mensual y anual. El 
fundamento para escoger el uno o el otro está en la facilidad de aplicación del objetivo estratégico, es decir se estableció de forma anual la consecución de objetivos que requieren algún tiempo considerable para su aplicación.

Tabla 7. Indicadores de gestión para la empresa del sector metalmecánico, perspectiva financiera

\begin{tabular}{|c|c|c|c|c|}
\hline Perspectiva & Objetivo estratégico & Indicador & Cuantificador & $\begin{array}{c}\text { Frecuencia } \\
\text { de } \\
\text { monitoreo }\end{array}$ \\
\hline \multirow{2}{*}{ Financiera } & $\begin{array}{c}\text { Disminuir los costos de } \\
\text { producción }\end{array}$ & $\begin{array}{c}\text { Margen } \\
\text { operativo }\end{array}$ & $\begin{array}{c}\text { (Cantidad gastada/Cantidad } \\
\text { presupuestada) } \times 100\end{array}$ & Mensual \\
\cline { 2 - 5 } & $\begin{array}{c}\text { Tecnificar el sistema de } \\
\text { cobranzas }\end{array}$ & $\begin{array}{c}\text { \% Nivel de } \\
\text { cumplimiento }\end{array}$ & $\begin{array}{c}\text { realizadas/No de actividades } \\
\text { programadas }) \times 100\end{array}$ & Anual \\
\cline { 2 - 5 } & $\begin{array}{c}\text { Incrementar la } \\
\text { rentabilidad }\end{array}$ & Índice de \\
rentabilidad & (Gastos netos/Total \\
facturado) $\times 100$ & Mensual \\
\hline
\end{tabular}

Tabla 8. Indicadores de gestión para la empresa del sector metalmecánico, perspectiva del cliente

\begin{tabular}{|c|c|c|c|c|}
\hline Perspectiva & Objetivo estratégico & Indicador & Cuantificador & $\begin{array}{c}\text { Frecuencia } \\
\text { de } \\
\text { monitoreo }\end{array}$ \\
\hline \multirow{2}{*}{ Del cliente } & $\begin{array}{c}\text { Incrementar el portafolio } \\
\text { de clientes }\end{array}$ & $\begin{array}{c}\text { Cartera de } \\
\text { clientes }\end{array}$ & $\begin{array}{c}\text { (Clientes nuevos/Total de } \\
\text { clientes) } \times 100\end{array}$ & Mensual \\
\cline { 2 - 6 } & $\begin{array}{c}\text { Acrecentar la satisfacción } \\
\text { del cliente }\end{array}$ & $\begin{array}{c}\text { Calidad de } \\
\text { servicio }\end{array}$ & $\begin{array}{c}\text { (№ de trabajos entregados/№ } \\
\text { de clientes satisfechos) } \times 100\end{array}$ & Mensual \\
\hline
\end{tabular}

Tabla 9. Indicadores de gestión para la empresa del sector metalmecánico, perspectiva de los procesos

\begin{tabular}{|c|c|c|c|c|}
\hline Perspectiva & Objetivo estratégico & Indicador & Cuantificador & $\begin{array}{c}\text { Frecuencia } \\
\text { de } \\
\text { monitoreo }\end{array}$ \\
\hline \multirow{3}{*}{$\begin{array}{l}\text { De los } \\
\text { Procesos } \\
\text { internos }\end{array}$} & $\begin{array}{l}\text { Establecer calidad en } \\
\text { los procesos }\end{array}$ & $\begin{array}{c}\text { \% } \\
\text { implementación } \\
\text { de la gestión } \\
\text { integral por } \\
\text { calidad } \\
\end{array}$ & $\begin{array}{c}\left(\mathrm{N}^{\circ} \text { de actividades }\right. \\
\text { realizadas } / \mathrm{N}^{\circ} \text { de } \\
\text { actividades programadas }) \mathrm{x} \\
100\end{array}$ & Anual \\
\hline & $\begin{array}{l}\text { Controlar el tiempo de } \\
\text { entrega del producto }\end{array}$ & $\begin{array}{c}\% \text { de } \\
\text { cumplimiento } \\
\text { de los tiempos } \\
\text { de entrega } \\
\end{array}$ & $\begin{array}{c}\text { (tiempo planificado/tiempo } \\
\text { real) } \times 100\end{array}$ & Mensual \\
\hline & $\begin{array}{l}\text { Estandarización de } \\
\text { procesos }\end{array}$ & $\begin{array}{c}\text { \% de } \\
\text { implementación } \\
\text { de } \\
\text { procedimientos }\end{array}$ & $\begin{array}{l}\left(\mathrm{N}^{\circ} \text { de procedimientos }\right. \\
\text { utilizados } / \mathrm{N}^{\circ} \text { procesos } \\
\text { existentes }) \times 100\end{array}$ & Mensual \\
\hline
\end{tabular}

Con el empleo del Cuadro de Mando Integral como herramienta de control de la gestión, se logró establecer indicadores y objetivos de acuerdo a la realidad que vive el sector metalmecánico, de manera que organizaciones de este tipo podrán mejorar la manera de manejar las empresas, se aplicó e interrelacionó la estrategia con las perspectivas financieras, del cliente, de los procesos 
internos y se consiguió el crecimiento y desarrollo dentro de un mercado competitivo y globalizado que exige cambios e innovación permanente.

Tabla 10. Indicadores de gestión para la empresa del sector metalmecánico, perspectiva de formación y crecimiento

\begin{tabular}{|c|c|c|c|c|}
\hline Perspectiva & $\begin{array}{c}\text { Objetivo } \\
\text { estratégico }\end{array}$ & Indicador & Cuantificador & $\begin{array}{c}\text { Frecuencia } \\
\text { de } \\
\text { monitoreo }\end{array}$ \\
\hline $\begin{array}{c}\text { De } \\
\text { Formación } \\
\begin{array}{c}\text { y } \\
\text { Crecimiento }\end{array}\end{array}$ & $\begin{array}{c}\text { Diseño de productos } \\
\text { y/o procesos } \\
\text { innovadores }\end{array}$ & $\begin{array}{c}\text { \% Nivel de } \\
\text { Innovación }\end{array}$ & $\begin{array}{c}\text { Generar planes de } \\
\text { innovadores/ } / \mathrm{N}^{\circ} \text { total de } \\
\text { productos }) \times 100\end{array}$ & Anual \\
\cline { 2 - 5 } & $\begin{array}{c}\text { capacitación } \\
\text { cumplimiento del de } \\
\text { adiestramiento }\end{array}$ & $\begin{array}{c}\mathrm{N}^{\circ} \text { de cursos realizados } / \mathrm{N}^{\circ} \\
\text { de cursos programados }) \times 100\end{array}$ & Anual \\
\hline
\end{tabular}

\section{Conclusiones y Recomendaciones}

\subsection{Conclusiones}

Las pymes estudiadas no contaban con una planificación estratégica debidamente estructurada; la propuesta de misión, visión, valores corporativos y objetivos estratégicos servirán como guía para futuros estudios para cada metalmecánica en forma particular.

Se logró establecer 10 indicadores de gestión, de acuerdo a los objetivos estratégicos trazados. En la perspectiva financiera se tomó en cuenta el concepto de ingresos y gastos para reducir los costos de producción y generar mayor rentabilidad, así como la tecnificación del sistema de cobros. En el enfoque del cliente se buscó ampliar la cartera de clientes y acrecentar su grado de satisfacción. En lo que respecta a los procesos internos se determinó la necesidad de estandarizar procesos, establecer planes de calidad y gracias a esto disminuir los tiempos de entrega de los productos. Finalmente en la perspectiva de aprendizaje y crecimiento se consideró el apoyo a la innovación como algo vital, así como la capacitación permanente del personal.

El Cuadro de Mando Integral permite a los miembros de las empresas estar alineados con la estrategia, de manera que sea posible dirigir su comportamiento y evaluar los logros al emplear indicadores de gestión.

La estructura organizacional carente de un estricto orden jerárquico, brinda oportunidades de participación plena a todos los miembros de la empresa y contribuye a la conformación de un buen clima laboral, que pudo ser percibido dentro de las empresas, esto facilitará la implantación de la estrategia y su control mediante el sistema de indicadores propuesta en el presente estudio.

El análisis DAFO posibilitó la identificación de las principales fortalezas de las empresas e indicó que poseen estructura flexible y totalmente autónoma, además la atención a los clientes se da de forma personalizada, se atiende todo tipo de requerimientos y existe facilidades de pago para los 
clientes. Como amenazas se destaca la presencia de grandes empresas con tecnología avanzada y la poca oferta de capacitaciones en el área metalmecánica y en temas como el control de gestión.

Estandarizar los procesos y capacitar a los trabajadores de acuerdo a las necesidades de cada operación va a garantizar el pleno establecimiento de roles específicos y responsabilidades dentro de las empresas.

\subsection{Recomendaciones}

Analizar y revisar el sistema de indicadores de gestión a fin de que éste se adecúe a las necesidades particulares de cada empresa metalmecánica.

Llevar a cabo campañas internas para difundir la misión, la visión, los valores y los principios de la empresa a todos los miembros de la organización. La efectividad de aplicar indicadores a los objetivos de una organización, requiere principalmente de la colaboración y trabajo en equipo de todos los miembros, cada trabajador debe comprender claramente los objetivos, las estrategias y definir su contribución y aporte al cumplimiento de los mismos.

Realizar un seguimiento constante a los resultados obtenidos con el objeto de evaluar el comportamiento de la organización y ejecutar una toma de decisión oportuna y acertada ante las desviaciones presentadas.

Invertir en maquinaria de última tecnología ya que esto permitirá que la empresa realice trabajos innovadores y esto ayudará también a que sus clientes publiciten los talleres, al resaltar la tecnología que poseen.

Validar el sistema de indicadores de gestión en las pymes metalmecánicas mediante el uso de herramientas informáticas.

\section{Bibliografía}

Castañeda, L. (2009). Alta dirección en las PYMES. México: Ediciones Poder.

CORPEI. (2009). Perfil de Metalmecánica. Recuperado el 19 de Noviembre de 2012, de PUCE: http://www.puce.edu.ec/documentos/perfil_de_metalmecanica_2009.pdf

Corporación EKOS. (2010). EKOS, Pymes 2010. PYMES, La pequeña y mediana empresa en el Ecuador, 14-31.

EKOS Negocios. (2012). PYMES: Contribución clave en la economía. Recuperado el 21 de Noviembre de 2012, de Revista EKOS: http://www.ekosnegocios.com/revista/pdfTemas/523.pdf

El Comercio. (2012). El sector de la metalmecánica crece a un ritmo moderado . Quito: El Comercio. 
Heredia, J. A. (2001). Sistema de indicadores para la mejora y el control integrado de la calidad de los procesos. Castellón de la Plana: Athenea.

Kaplan, R., \& Norton, D. (2009). El cuadro de mando integral. Barcelona: Brosmac.S.L.

Marquéz, E. (2006). Diseño de un sistema de indicadores de gestión de una empresa metalmecánica basado en el modelo del cuadro de mando integral. Barquisimeto: Universidad Nacional Experimental Politécnica "Antonio José de Sucre".

Reyes, L. (2011, julio 7). Estadística, matemática y computación. Recuperado Marzo 18, 2012, de reyesestadística.blogspot: http://reyesestadistica.blogspot.com/2011/07/muestreo-simplealeatorio.html

Scali, J., \& Tapia, G. (2011). Tablero de Comando en las PyMEs. Buenos Aires: Omicron.

Universidad Andina Simón Bolívar. (20 de Diciembre de 2012). Pymes pueden vender más a 11 países. Recuperado el 4 de Enero de 2013, de Observatorio de la pequeña y mediana empresa:

http://www.uasb.edu.ec/contenido_centro_programa_noticia_cont.php?cd=4351\&cd_centro= $15 \& \mathrm{sw}=\mathrm{ev}$ 